\title{
In-Flight Wing Deformation Measurement System for Small Unmanned Aerial Vehicles
}

\author{
Zi Yang Pang, ${ }^{*}$ Carlos E. S. Cesnik ${ }^{\dagger}$ and Ella M. Atkins \\ The University of Michigan, Ann Arbor, MI, 48109-2410
}

\begin{abstract}
This paper presents a new method to measure wing deflections in flight for small UAVs. It employs a pair of high resolution stereo cameras and LED wing markers, as well as a small form factor computer for control and storage. Post-processing of all the data is done off-line. Accuracy benchmark tests are conducted. Finally, theoretical discussion of wing shape reconstruction is presented. The method employs numerical optimization by minimizing the difference between numerical geometrically nonlinear slender beam equations and observed markers points with associated uncertainties.
\end{abstract}

\section{Nomenclature}

$\begin{array}{ll}c_{x}, c_{y} & =\text { principal focal point } \\ C & =\text { circularity } \\ \mathrm{d} & =\text { disparity } \\ e & =\text { error metric } \\ f_{x}, f_{y} & =\text { focal length (in pixels) } \\ \mathrm{F} & =\text { focal length } \\ \boldsymbol{F}_{i}, \boldsymbol{M}_{i} & =\text { design variables (point forces and moments in UM/NAST) } \\ J & =\text { cost functional } \\ k_{l}, k_{2}, k_{3} & =\text { radial distortion parameters } \\ k & =\text { nodal points where point forces and moments are applied } \\ N & =\text { number of marker points } \\ p_{l}, p_{2} & =\text { tangential distortion parameter } \\ \mathbf{p}_{\mathrm{i}} & =\text { beam deflection computed by UM/NAST } \\ \mathbf{q}_{\mathrm{i}} & =\text { measured beam deflection } \\ \mathbf{Q} & =\text { reprojection matrix } \\ r & =\text { radial distance } \\ \mathbf{R} & =\text { rotation matrix } \\ \mathbf{t} & =\text { translational vector } \\ T_{x} & =x \text { component of rotation matrix } \\ x, y & =\text { image coordinates (distorted) } \\ x_{c}, y_{c} & =\text { image coordinates (pin hole model) } \\ X, Y, Z & =\text { physical coordinates } \\ \mathbf{X}_{\mathrm{cb}, \mathrm{i}} & =\text { known checkerboard coordinates } \\ \sigma_{i} & =\text { error weight }\end{array}$

\section{Introduction}

$\mathrm{M}$ odern high altitude long endurance (HALE) aircraft is characterized by high aspect ratio wings and, if present, thin fuselage. Coupled with lightweight construction, HALE aircraft tend to be very flexible, exhibiting large

\footnotetext{
* PhD Pre Candidate (pziyang@umich.edu), Department of Aerospace Engineering, Member, AIAA

† Professor (cesnik@ umich.edu), Department of Aerospace Engineering, Fellow, AIAA

*Associate Professor (ematkins@ umich.edu), Department of Aerospace Engineering, Associate Fellow, AIAA
} 
wing deformation at normal operating loads. As a result, unlike traditional aircraft, it requires a nonlinear aeroelastic design methodology. However, this is still an emerging field with limited predictive capabilities (e.g., Helios accident ${ }^{1}$ ).

At University of Michigan's Active Aeroelasticity and Structures Research Laboratory, much research is being conducted to remedy this shortfall. On the theoretical/numerical front, the University of Michigan Nonlinear Aeroelastic Simulation Toolbox (UM/NAST) ${ }^{2}$ has been developed. It is a framework capable of simulating coupled nonlinear aeroelasticity and flight dynamics of very flexible aircraft. On the experimental front, an aeroelastic test vehicle named X-HALE ${ }^{3}$ (see Figure 1) has been designed and built to collect nonlinear aeroelastic data. The objective is to increase understanding of aeroelastic behavior through flight trials of flexible aircraft. In addition, correlation of theoretical with experimental results is done to improve existing numerical tools. Though X-HALE has been instrumented with an inertia measurement unit (IMU) to measure center body vehicle response, its intended strain-gage-based wing shape measurement system has proved unfeasible in the field. For X-HALE in specific, but for that class of unmanned aerial vehicles (UAV) in general, a light-weight, low-power, compact measurement system for wing shape is needed.

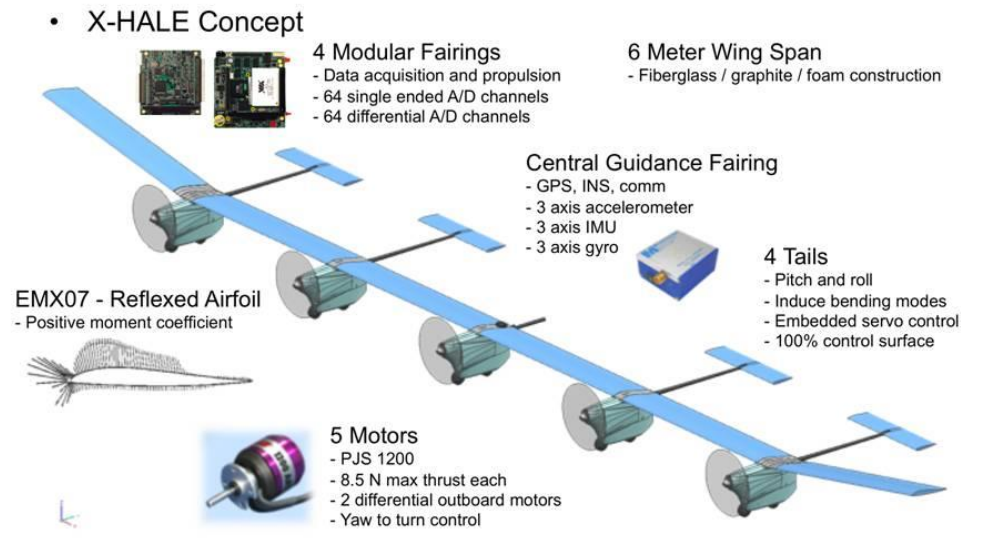

Figure 1. UM X-HALE 6-meters aeroelastic test vehicle model

Vision-based measurement technique is an attractive option to measure wing deformation in flight. Advances in computer vision technologies led to a rapid growth in vision measurement systems. Wide spread adoption of $\mathrm{CCD} / \mathrm{CMOS}$ cameras for general surveillance and image capture (e.g, CCTV, webcams) resulted in rapid development of camera technology, culminating in increasingly better camera/sensor specification, as well as miniaturization. Thus, high resolution compact CCD cameras are widely available from different vendors, e.g., Pointgrey, ${ }^{*}$ IDS, ${ }^{\dagger}$ and Ximea. ${ }^{\ddagger}$ By and large they are lightweight and compact. Many of the applications found in literature spans diverse fields such as human kinesiology, ${ }^{4}$ robotic navigation, ${ }^{5}$ and scene reconstruction. ${ }^{6}$ Aerospace researchers have also employed vision based methods as it is non-intrusive measurement method, ideal for situations which require interference to the flow to be avoided. Hence, it is not surprising to find application in shape deformation or model attitude measurements for wind tunnel tests. Video Model Deformation ${ }^{7}$ (VMD) and Projection Moiré Interferometry (PMI), stereo-optical Recovery of Attitude and Deformation by Crossed Anamorphoses (RADAC) ${ }^{8}$ and Visual Image Correlation ${ }^{9}$ (VIC) system for miniature aerial vehicle (MAV) wind tunnel test are some examples. Burner et al. ${ }^{10}$ provide a comparison between some of the above mentioned system.

Unfortunately, in-situ flight vision measurement systems are extremely rare. The earliest experiment is probably HiMAT Aeroelastic Tailored Wing study commissioned by NASA in 1980s. ${ }^{11}$ In that study, infrared light emitting diodes (LED) were mounted in aerodynamically shaped fixtures on the wing. They were focused and captured using a light sensitive diode array mounted on the fuselage. The wing deformation was recovered by comparing the image with a calibration of known displacement. A similar system was employed by the Active Aeroelastic Wing study on a modified F/A-18. ${ }^{12}$ More recently, a JAXA Beechcraft Queen Air low wing research aircraft was instrumented with a stereovision rig. ${ }^{13}$ The setup included two high resolution CCD cameras looking out through its cabin windows, capturing fiducial markers painted on the wing. The images were post-processed with a pin-hole camera

\footnotetext{
${ }^{*}$ http://ww2.ptgrey.com/

$\dagger$ http://en.ids-imaging.com/

\#ttp://www.ximea.com/
} 
calibration model and resultant wing bending and twist angles were successfully recovered. However, from published literature, no vision measurement system has been used in small UAV.

Another promising deformation measurement type found in literature is indirect shape estimation techniques using strain measurements, which can come from foil gauges ${ }^{14}$ and/or fiber-optics Bragg gratings (FBG). ${ }^{15,16}$ External perturbations in strain affect the resistance (foil gauges) and optical properties (FBG), respectively. By accurately measuring those, the change in strain can be recovered. This is subsequently converted to displacements using various reconstruction techniques ${ }^{17}$. Two examples of FBG-based system from NASA ${ }^{18,19}$ have been applied to flying UAVs, as summarized in Table 1. Of the two, only the one may be fit for small UAV applications when comparing to X-HALE class application.

Table 1. Comparison of UAV wing measurement systems

\begin{tabular}{cccc}
\hline \hline Parameter & $\begin{array}{c}\text { X-HALE } \\
\text { Requirement }\end{array}$ & FBG UAV $^{18}$ & FBG Predator $^{19}$ \\
\hline \hline Weight $(\mathrm{kg})$ & 1.4 & 1.3 & 10.4 \\
Dimensions $(\mathrm{mm})$ & $115 \times 165 \times 30$ & $177 \times 152 \times 127$ & $191 \times 330 \times 330$ \\
Power $(\mathrm{W})$ & 90 & 10 & 112 \\
Sample Rate $(\mathrm{Hz})$ & 30 & 0.5 & $50(2$ fibers $)$ \\
\hline
\end{tabular}

Table 2 attempts to rank general performance metrics of each measurement type, although the exact implementation details may affect this ranking. As one can see, the strain-gauge based system has serious limitation on data quality and noise rejection in the field, and has been eliminated from further considerations. On the other hand, FBG based system has excellent performance on those metrics, but current commercial implementations are too heavy and too sizable for small UAV applications. Therefore, this paper aims to present a vision-based measurement system which is small and lightweight and can be carried by a small UAV. This will be useful for researchers interested in measuring aeroelastic behavior of small flexible aircraft and is being integrated in the XHALE for future flight test.

Table 2. Relative performance metrics for various shape measurement systems

\begin{tabular}{cccc}
\hline \hline Performance Measure & Vision-based & Strain Gauge Based & FBG Based \\
\hline \hline Weight and Volume Requirement & + & ++ & - \\
Power Consumption & + & ++ & + \\
Data Quality & ++ & - & ++ \\
Noise Rejection & + & -- & ++ \\
\hline \multicolumn{4}{c}{ Key: ++ (very good), +(good), - (bad) }
\end{tabular}

\section{Theoretical Formulation}

In what follows, the fundamental theoretical background related to vision measurements is presented, along with the shape recovery process for geometrically nonlinear deformations.

\section{A. Pinhole Camera Model}

Let $Q_{i}$ denotes the $i$-th point in the physical space with coordinates $\left(X_{i}, Y_{i}, Z_{i}\right)$ and $q_{i}$ denotes a point in the image space with coordinates $\left(x_{i}, y_{i}\right)$, and in homogenous coordinates, $q_{i}=\left(x_{i}, y_{i}, 1\right)$. The projective transform, as the linear map from $q_{i}$ to $Q_{i}$, can be expressed as ${ }^{20}$

$$
s\left[\begin{array}{c}
x \\
y \\
1
\end{array}\right]=\left[\begin{array}{ccc}
f_{x} & 0 & c_{x} \\
0 & f_{y} & c_{y} \\
0 & 0 & 1
\end{array}\right]\left[\begin{array}{ll}
\mathbf{R} & \mathbf{t}
\end{array}\right]\left[\begin{array}{c}
X \\
Y \\
Z \\
1
\end{array}\right]
$$

where $f_{x}, f_{y}, c_{x}, c_{y}$ are camera instrinsic coefficients, $\mathbf{R}$ is a $3 \times 3$ rotation matrix, $\mathbf{t}$ is a $3 \times 1$ camera translation vector, and $s$ is an arbritrary scale factor. Due to the presence of the lens, there exist two forms of distortion: radial 
and tangential. To correct for lens distortion, the points on the image plane as produced by a perfect pin hole camera $\left(x_{c}, y_{c}\right)$ can be expressed as ${ }^{21}$

$$
\begin{aligned}
& x_{c}=x\left(1+k_{1} r^{2}+k_{2} r^{4}+k_{3} r^{6}\right)+\left[2 p_{1} x y+p_{2}\left(r^{2}+2 x^{2}\right)\right] \\
& y_{c}=y\left(1+k_{1} r^{2}+k_{2} r^{4}+k_{3} r^{6}\right)+\left[p_{1}\left(r^{2}+2 y^{2}\right)+2 p_{2} x y\right] \\
& r^{2}=x^{2}+y^{2}
\end{aligned}
$$

Therefore, there are five distortion coefficients $k_{1}, k_{2}, k_{3}, p_{1}$ and $p_{2}$ and four intrinsic camera parameters that must be obtained in order to transform between $q_{i}$ and $Q_{i}$. This is done in the camera calibration step presented in the section below.

\section{B. Camera Calibration}

All vision processing employed in this study is done using openCV, ${ }^{*}$ which is an open source computer vision library. It is available under the BSD license. ${ }^{\dagger}$ Bradiski and Kaehle ${ }^{22}$ provides detailed theory and implementation of OpenCV's algorithm. For calibration, the software employs a calibration method outlined by Zhang. ${ }^{20}$ A series of checkerboard images at different poses is used to calculate the 9 intrinsic parameters (mentioned above) using nonlinear optimization.

\section{Marker Detection}

The aperture, gain and shutter speed are controlled to obtain images of active markers as bright circular orbs on dark background. This is done to aid background rejection in image processing. Brightness thresholding is used to convert the greyscale image to a black and white image. Each filled contour represents a LED marker and the centroid can be easily found. Built-in OpenCV function "blobdetector" is used for this purpose.

During software post processing, a filled contour is first checked for circularity,

$$
C=4 \pi \frac{\text { marker area }}{\text { marker perimeter }}
$$

and is only admitted as a legitimate marker candidate if $C>0.8$.

\section{Stereo Imaging}

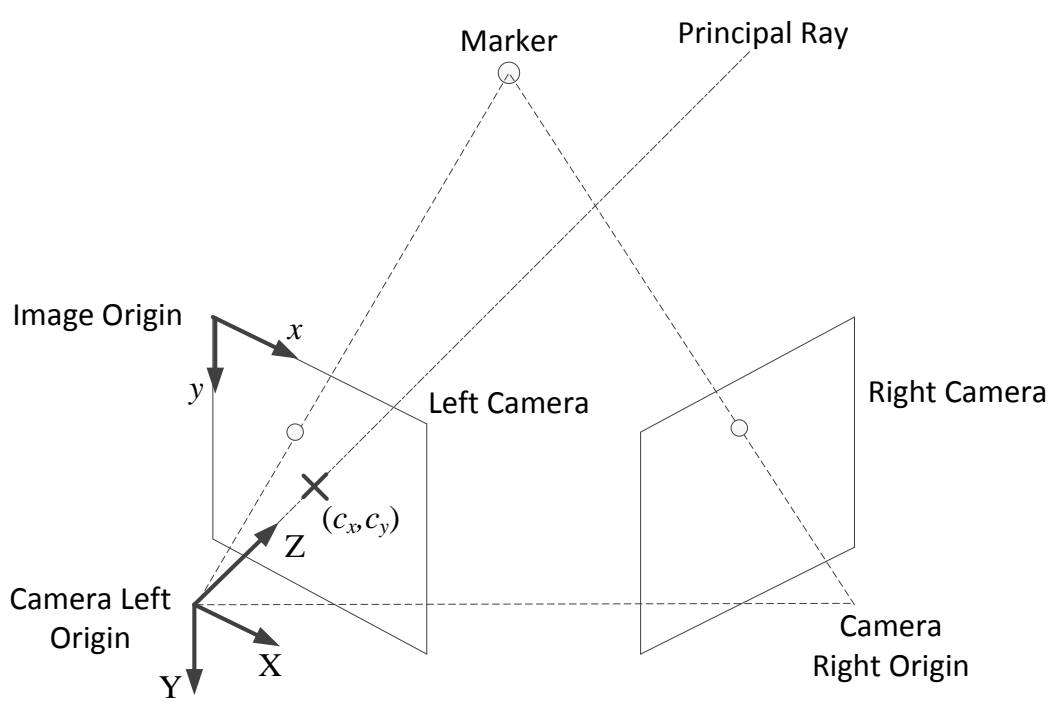

Figure 2. Schematic of stereo camera setup

\footnotetext{
*http://opencv.org/

${ }^{\dagger}$ http://opensource.org/licenses/BSD-3-Clause
}

American Institute of Aeronautics and Astronautics 
The calibration images captured by the left and right cameras (Figure 2) are first undistorted using distortion coefficients from the calibration step. Stereocalibration to relate the pose of the left and right cameras is then performed using Leven-Marquardt optimization ${ }^{23}$ in OpenCV. Next, stereo rectification using Bouget's algorithm* is applied and this step produces images which are frontal.

Using the rectified images, image correspondence is performed. This process matches the features from the left and right images to create a disparity map. Correspondence is done via a simple sorting of markers by vertical position, where the furthest markers are lowest on the image (by convention, in each image, the pixel location is defined with the origin at the top left corner).

Subsequently, using the coordinates of the matched marker on left and right images, triangulation is performed to produce a depth map. The reprojection matrix $\mathbf{Q}$ is defined by

$$
\mathbf{Q}=\left[\begin{array}{cccc}
1 & 0 & 0 & -c_{x} \\
0 & 1 & 0 & -c_{y} \\
0 & 0 & 0 & f \\
0 & 0 & -T_{x} & \left(c_{x}-c_{x}{ }^{r i g h t}\right) / T_{x}
\end{array}\right]
$$

and

$$
\mathbf{Q}\left[\begin{array}{c}
x \\
y \\
d \\
1
\end{array}\right]=\left[\begin{array}{c}
X \\
Y \\
Z \\
W
\end{array}\right]
$$

where all parameters are obtained from the left camera unless otherwise stated and it is also used to hold the reference coordinate system. Physical coordinates can be recovered from the homogenous coordinates by

$$
\begin{aligned}
& X=X / W \\
& Y=Y / W \\
& Z=Z / W
\end{aligned}
$$

Figure 3 summarizes the workflow of the stereovision algorithm.

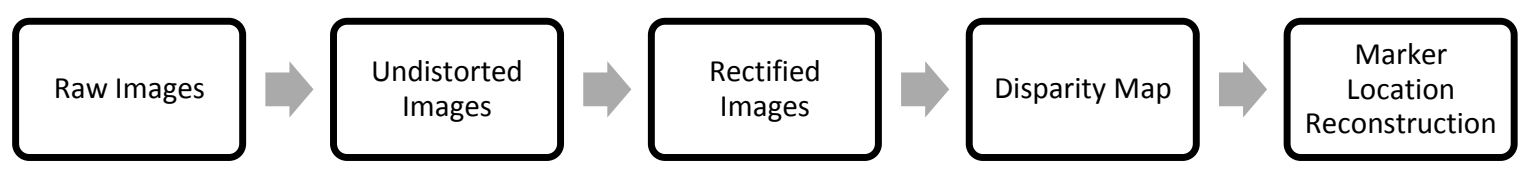

Figure 3. Stereo imaging workflow

\section{E. 3-D Wing Shape Reconstruction}

High aspect ratio wings are expected to experience geometrically nonlinear deformation in bending (out of plane and in plane) and torsion, but minimal chordwise deformation. Therefore, the wing can be modeled using a geometrically nonlinear beam, which is accomplished here using UM/NAST ${ }^{24} \mathrm{UM} / \mathrm{NAST}$ is an aeroelastic solver that employs a strain-based finite element beam formulation and is capable of solving aeroelastic nonlinear slender structure problems. Part of it is a static module that solves the beam deflection for given loads. This is the module which is used for the wing reconstruction detailed below.

Now, let point forces and moments be applied at $k$ selected nodal points. By varying the magnitude of the applied force/moments, a particular beam shape can be obtained, provided $k$ is sufficiently dense.

Let $\mathbf{q}_{i}$ be the observation coordinates recovered by the above stereovision setup. From the calibration process, an estimate of the uncertainty associated with a given marker can be obtained. By adjusting the forces and moments,

${ }^{*}$ http://www.vision.caltech.edu/bouguetj/calib_doc/

American Institute of Aeronautics and Astronautics 
the Euclidean difference between observed $\mathbf{q}_{i}$ and computed beam deflection $\mathbf{p}_{i}$ can be minimized. This can be formulated as a optimization problem by minimizing the cost functional

$$
J=\frac{1}{N} \sum_{i=1}^{N} \frac{\left|\mathbf{q}_{i}-\mathbf{p}_{i}\right|^{2}}{\sigma_{i}^{2}}
$$

where $N$ is the number of observation points and $\sigma_{i}$ is the error weight associated with $i^{\text {th }}$ point. If the uncertainty of the observed point is high, its influence on the overall functional cost is low. The design variables are the set of point forces and moments $\left\{\mathbf{F}_{j}, \mathbf{M}_{j}\right\}$ at selected nodes, $j=1, \ldots, k$. The wing shape is therefore given by the solution from this optimization problem. The flow chart in Figure 4 details the solution workflow.

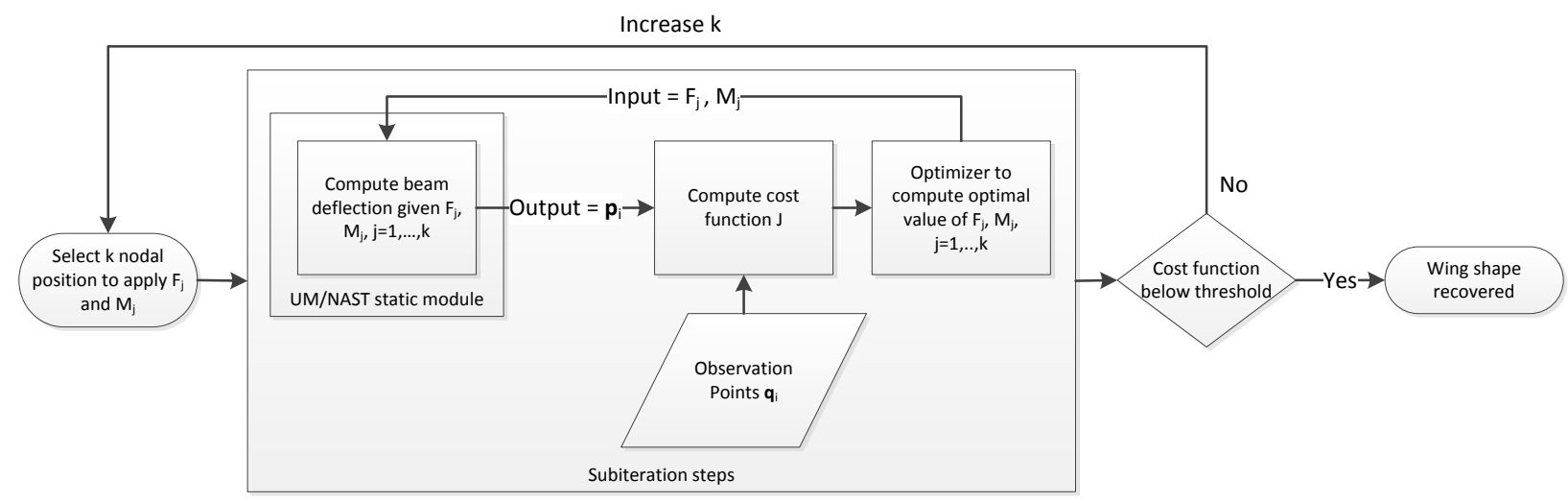

Figure 4. Shape recovery optimization problem for a given snapshot in time

\section{Stereo-vision Instrumentation Setup}

Description of the basic hardware/software setup developed based on the proposed approach and being implemented in the X-HALE is described next.

\section{A. Image Acquisition System}

Two pairs of cameras will be mounted on the center of X-HALE, looking towards the left and right wing, respectively. The overall schematic is shown in Figure 5, the distance between each camera pair is $279.4 \mathrm{~mm}$ wide and $80 \mathrm{~mm}$ tall. Pointgrey Flea3 FL3-U3-32S2M-CS ${ }^{*}$ cameras with Kowa LMVZ3510-IR ${ }^{\dagger}$ lens are used for video capture. The camera is a monochrome model with resolution up to 2080x1552 at 60 frames per second (FPS). The varifocal Kowa lens allows manual setting of aperture, zoom and focal point. It is vital that images taken by the cameras are synchronized in the stereovision measurements. All four cameras are synchronized using hardware

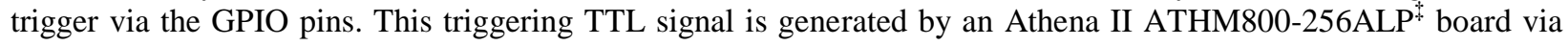
single digital I/O channel that will be used on-board of the aircraft.

The resulting video streams are sent via USB 3.0 protocol to the EPIC form-factor IEI NANO QM770 Single Board Computer ${ }^{\S}$ (SBC). Due to the large bandwidth of video data, solid state drives (SSD) were selected to be the storage medium. The video data from each pair of cameras is written to a 512 GB SSD. The operating system is Ubuntu 12.04.3 LTS.* $^{* *}$ The architecture is depicted in Figure 6 and the mounting location depicted in Figure 7. Table 3 summarizes the overall weight, power, and volume properties of this setup.

\footnotetext{
*http://ww2.ptgrey.com/USB3/Flea3

${ }^{\dagger}$ http://kowa.eu/cctv/en/LMVZ3510-IR.php

$*$ http://www.diamondsystems.com/products/athenaii

$\S$ http://www.ieiworld.com/index.aspx

**tp://www.ubuntu.com
} 


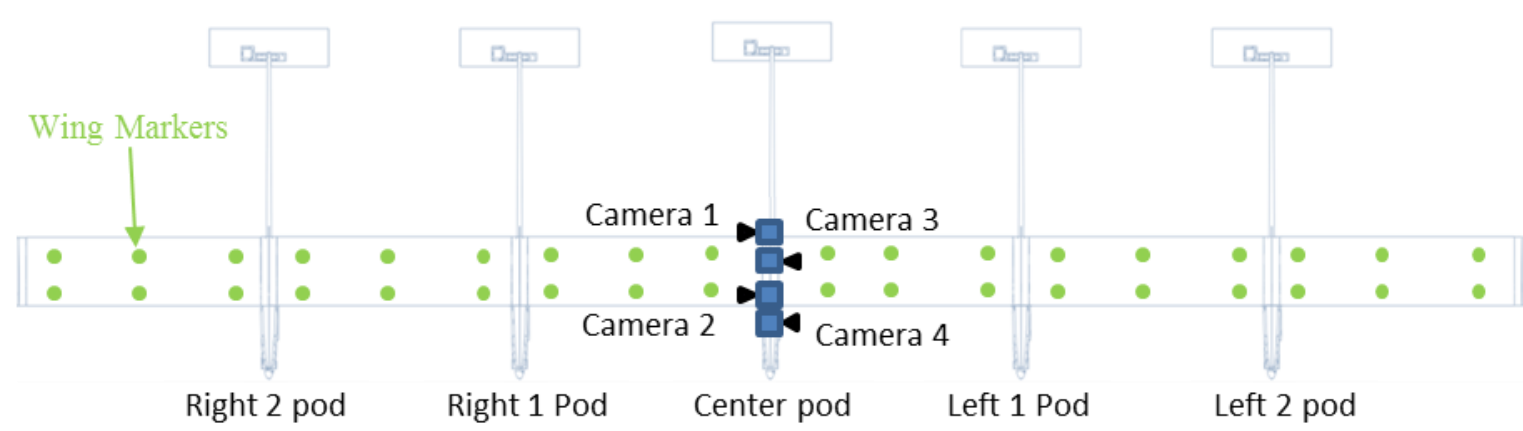

Figure 5. Vision measurement system mounted on X-HALE (top down view)

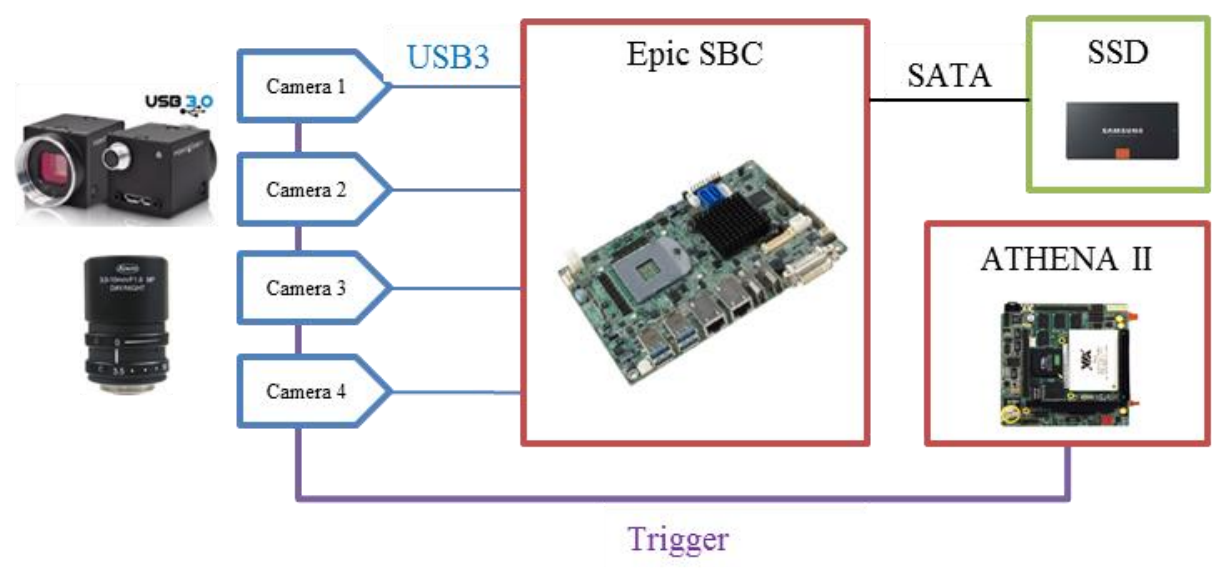

Figure 6. Camera system architecture

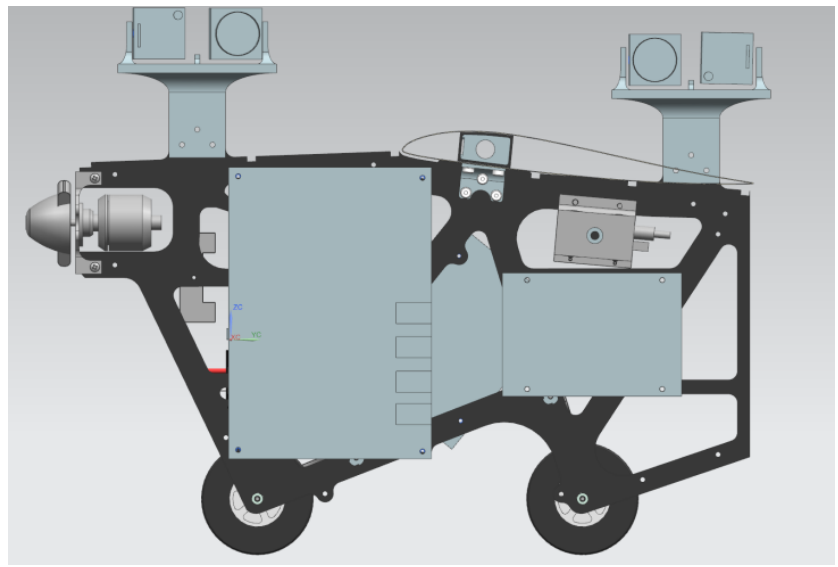

Figure 7. Camera frame mount

Table 3. Image acquisition system parameters for setup shown in Figure 6

\begin{tabular}{cc}
\hline Total weight & $\sim 1400 \mathrm{~g}$ \\
Power & $<90 \mathrm{~W}(60 \mathrm{~W}$ used for NANO) \\
Voltage & $12 \mathrm{~V}$ (NANO) \\
& $5 \mathrm{~V}$ (Athena II \& cameras) \\
Dimensions & $115 \mathrm{~mm} \times 165 \mathrm{~mm} \times 16.5 \mathrm{~mm}$ (NANO) \\
& $100 \mathrm{~mm} \times 35 \mathrm{~mm} \times 30 \mathrm{~mm}$ (camera+lens) \\
\hline
\end{tabular}


The Athena II is also responsible for the collection of other analog instrumentation on X-HALE. To ensure time synchronization between the computer systems, Precision Time Protocol (PTP) ${ }^{*}$ is ported to run on QNX6.5 (Athena II) and compiled on Ubuntu (NANO SBC), with the Athena II acting as the master clock. This setup is capable of achieving time synchronization of within $0.1 \mathrm{~ms}$.

\section{B. Target Markers}

10,000-mcd LED markers are mounted on the wing surface. Using high brightness LED, fast shutter speed can be employed to reduce motion blur. Also, it allows easy brightness thresholding during image processing. There will be three pairs of LED markers on each 1-meter segment of the wing, making a total of 36 markers on the entire XHALE (see Figure 5).

\section{Results and Discussion}

Laboratory tests have been conducted to verify and validate the procedure proposed in this paper.

\section{A. Camera Calibration and Stereoretification Results}

The cameras are mounted onto specially designed frames shown in Figure 7. These frames will eventually be integrated on the center pod of X-HALE. The image parameters (see

Table 4) are set via PointGrey Flycapture SDK. ${ }^{\dagger}$

Table 4. Imaging parameters

\begin{tabular}{ll}
\hline Image Resolution & $2080 \times 1552$ \\
Bitmap Depth & 8 -Bits Greyscale \\
Image Size & $3.22 \mathrm{MB}$ \\
Frame Rate & $25 \mathrm{~Hz}$ (Externally Triggered) \\
Throughput (1 Camera) & $80 \mathrm{MB} / \mathrm{s}$ \\
\hline
\end{tabular}
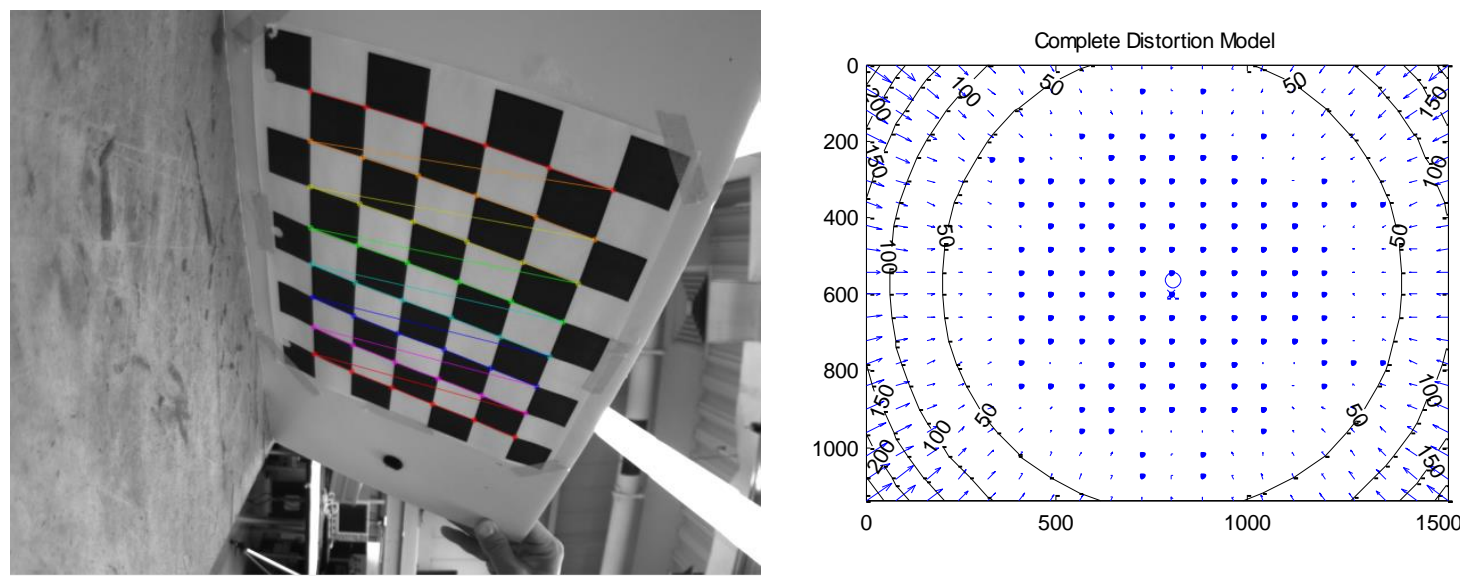

Figure 8. Camera calibration image (left); camera distortion model (right)

The lens setup is calibrated using the procedure outlined above. Fifteen views of a checkerboard were used to calibrate the intrinsic parameters independently (Figure 8) before being used to perform stereo calibration and rectification. Reprojection errors for different focal lengths are reported in Table 5. Note that although reported together, the left and right camera reprojection error are for intrinsic calibration while the stereo camera system reprojection error is for combined view. Therefore, the higher pixel error in the stereo camera system is expected and is well within desirable values. Selection of focal length is a tradeoff between field of view (to keep all markers

\footnotetext{
*http://ptpd.sourceforge.net/

${ }^{\dagger}$ http://ww2.ptgrey.com/sdk/flycap
} 
in view) and detection accuracy (of individual markers) as illustrated in Figure 9. A longer focal length results in the view of the displacement being "magnified," hence better accuracy.

Table 5. Reprojection error (in pixels) obtained from OpenCV calibration

\begin{tabular}{lccc}
\hline \hline Source & $\mathrm{F}=3.5 \mathrm{~mm}$ & $\mathrm{~F}=6.75 \mathrm{~mm}$ & $\mathrm{~F}=10 \mathrm{~mm}$ \\
\hline \hline Left Camera & 0.60 & 0.67 & 1.13 \\
Right Camera & 0.69 & 0.72 & 1.28 \\
\hline \hline Stereo Camera System & 0.70 & 0.73 & 1.42 \\
\hline
\end{tabular}
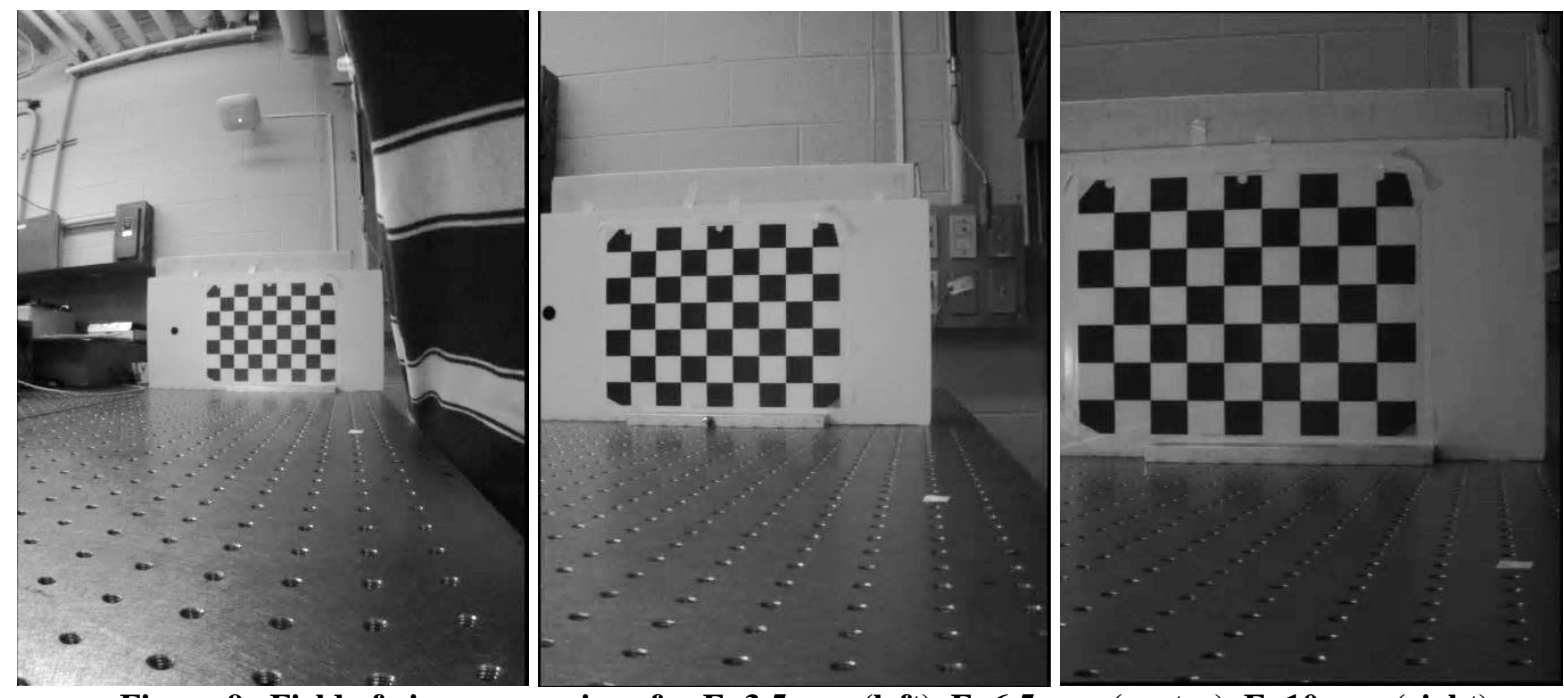

Figure 9. Field of view comparison for $F=3.5 \mathrm{~mm}$ (left), $\mathrm{F}=6.5 \mathrm{~mm}$ (center), $\mathrm{F}=10 \mathrm{~mm}$ (right)

\section{B. Benchmarking Results}

For benchmark accuracy tests, checkerboards are placed at predetermined distances (Z) away from the cameras. The physical coordinates (in object frame) are known. The error metric is defined to be the mean 2-norm of the difference between observed $\boldsymbol{q}_{i}$ and known coordinate $\boldsymbol{X}_{\boldsymbol{c} b, i}$ for $N$ checkerboard corners:

$$
e=\frac{1}{N} \sum_{i=1}^{N}\left\|\mathbf{q}_{i}-\mathbf{X}_{c b, i}\right\|_{2}
$$

As expected, Table 6 shows the error increases as the $\mathrm{Z}$ distance increases. This is due to the fact that a small change in displacement at nearer distances results in a larger disparity and hence, more accurate reconstruction. In addition, the error decreases as the focal length increases. Figure 10 (left) shows the reconstruction of the checkerboards in the left camera frame. It also shows that the reconstruction error is independent on its pixel location. Note that due to field of view issues, some results cannot be obtained and are noted by " - " in Table 6.

\section{Table 6. Error metric with checkerboard target for different focal lengths}

\begin{tabular}{ccccc}
\hline \hline Set & $\begin{array}{c}\text { Z Distance } \\
(\mathrm{mm})\end{array}$ & $\mathrm{F}=3.5 \mathrm{~mm}$ & $\mathrm{~F}=6.75 \mathrm{~mm}$ & $\mathrm{~F}=10 \mathrm{~mm}$ \\
\cline { 3 - 5 } & 500 & $-0.22 \pm 0.21$ & - & - \\
\hline 1 & 750 & $-0.34 \pm 0.25$ & - & - \\
2 & 1000 & $-0.99 \pm 0.49$ & $-0.4 \pm 0.28$ & - \\
3 & 1250 & $-1.55 \pm 0.76$ & $-1.4 \pm 0.67$ & - \\
4 & 1500 & $-2.30 \pm 1.12$ & $-2.5 \pm 1.15$ & $-0.90 \pm 0.48$ \\
5 & 1750 & $-2.88 \pm 1.37$ & $-3.5 \pm 1.59$ & $-1.98 \pm 0.91$ \\
6 & 2000 & $-3.42 \pm 1.68$ & $-4.4 \pm 2.04$ & $-2.82 \pm 1.30$ \\
7 & & & & \\
\hline
\end{tabular}



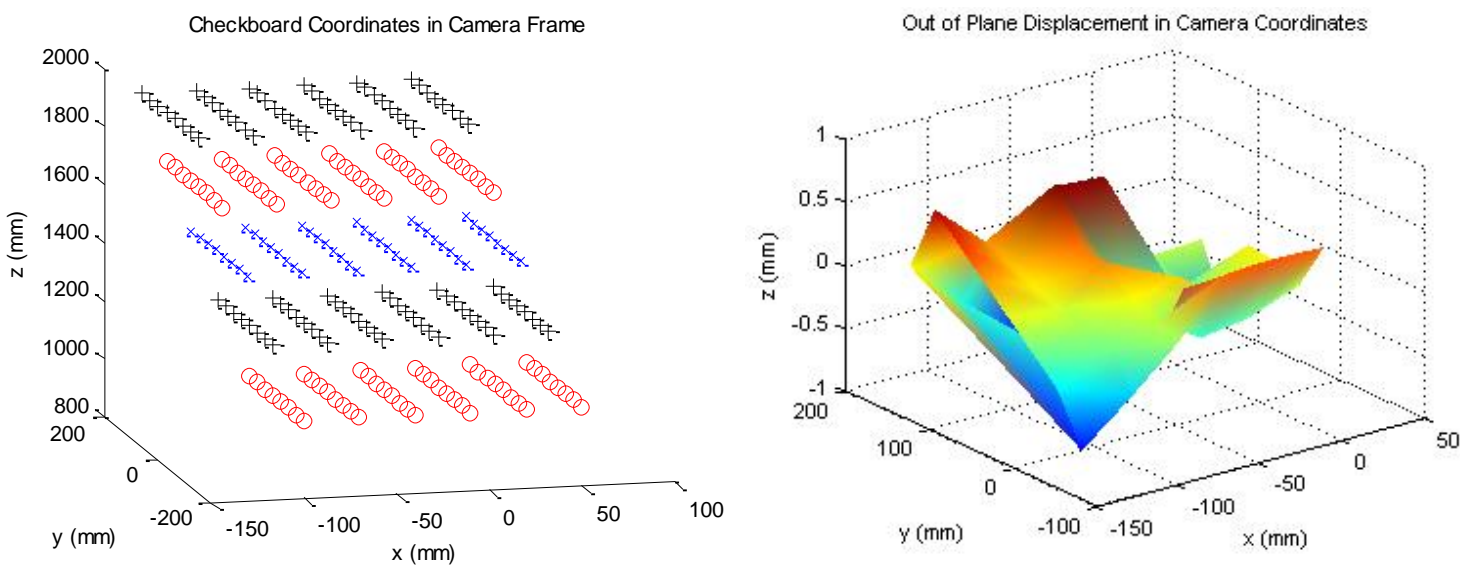

Figure 10. Recovered checkerboard coordinates $F=6.75 \mathrm{~mm}$ (left) out of plane displacement for checkerboard at $\mathrm{Z}=1000 \mathrm{~mm}, \mathrm{~F}=6.75 \mathrm{~mm}$ (right)

Subsequently, a LED marker pair was prototyped on a breadboard. The distance between the LEDs is $10.16 \mathrm{~mm}$. The same experiment was repeated to verify the performance of a LED marker against checkerboards. Figure 11 shows the marker centroid detection of a correctly exposed LED marker. It is very important to ensure the scene is not over-exposed. An over-exposed scene causes excessive "starry halo" around the LED marker, ruining a welldefined circular shape. Table 7 shows the accuracy of LED markers are similar to the checkerboards for $\mathrm{z}>1500$ $\mathrm{mm}$. The discrepancy at shorter distance is probably due to the "bulb" shape of the LED that no longer acts as a 2-D marker, effectively, and the centroid is "shifted."
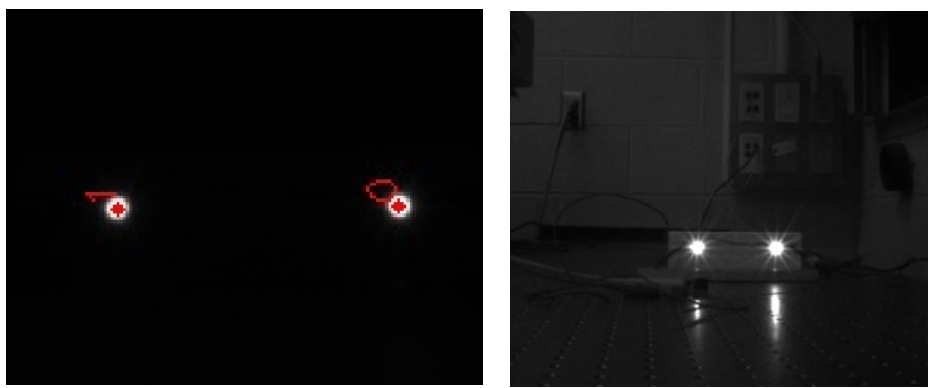

Figure 11. Centroid detection of LED markers with 2 ms exposure time (left); over-exposed image at 50 ms (right)

Table 7. Error metric with LED target for different focal length

\begin{tabular}{ccccc}
\hline \hline Set & $\begin{array}{c}\text { Z Distance } \\
(\mathrm{mm})\end{array}$ & $\mathrm{F}=3.5 \mathrm{~mm}$ & $\mathrm{~F}=6.75 \mathrm{~mm}$ & $\mathrm{~F}=10 \mathrm{~mm}$ \\
\cline { 4 - 6 } & 500 & -3.41 & - & - \\
2 & 750 & -3.86 & - & - \\
3 & 1000 & -4.29 & -4.07 & - \\
4 & 1250 & -4.78 & -4.83 & - \\
5 & 1500 & -5.19 & -5.61 & -4.02 \\
6 & 1750 & -5.61 & -6.40 & -4.76 \\
7 & 2000 & -6.27 & -7.13 & -5.52 \\
\hline
\end{tabular}

In addition, the performance of the EPIC SBC and the SSD were also benchmarked. Although the theoretical bandwidth limit of USB $3.0^{*}(450 \mathrm{MB} / \mathrm{s})$ and SSD sequential write ${ }^{\dagger}(520 \mathrm{MB} / \mathrm{s})$ are not exceeded, actual performance will be degraded due to operating system overhead. The bottleneck in either receiving (USB 3.0) or writing (SSD) will result in skipped frames. Table 8 shows the performance benchmark of four Flea3 cameras

\footnotetext{
* http://www.usb.org/developers/ssusb

${ }^{\dagger}$ http://www.samsung.com/us/computer/memory-storage/MZ-7PD256BW-specs
}

10

American Institute of Aeronautics and Astronautics 
recording simultaneously. The resolution is varied while keeping the frame rate at $25 \mathrm{~Hz}$ and 30,000 frames (approximately 20 minutes of video time) are captured. Currently, the software execution is monolithic, which is to say the capture and write to disk commands are run sequentially. In future generation of the software, multithreaded approach will be employed to separate into read and write threads. Also, ring buffers are currently implemented to reduce the number of dropped frames due to the SSD write latency.

Table 8. Hardware read-and-write performance

\begin{tabular}{|c|c|c|c|}
\hline $\begin{array}{c}\text { Image } \\
\text { Resolution }\end{array}$ & $\begin{array}{l}\text { Theoretical Image } \\
\text { Throughput (MB/s) }\end{array}$ & Write Type & \% Dropped Frames \\
\hline $2080 \times 1552$ & 322.82 & $\begin{array}{l}\text { fwrite, no buffer, no } \\
\text { threading }\end{array}$ & 3.83 \\
\hline $1920 \times 1080$ & 207.36 & $\begin{array}{l}\text { fwrite, no buffer, no } \\
\text { threading }\end{array}$ & 0.9 \\
\hline $1080 \times 720$ & 77.76 & $\begin{array}{c}\text { fwrite, no buffer, no } \\
\text { threading }\end{array}$ & 0.0 \\
\hline
\end{tabular}

\section{Functional Test in Outdoor Condition}

This test was conducted to verify the performance of the LED markers under bright direct sunlight. By decreasing the shutter speed, the markers were easily isolated from the background. Although the optimal shutter speed is dependent on ambient lighting condition, the order of magnitude is about $1-5 \mathrm{~ms}$ (Figure 12).

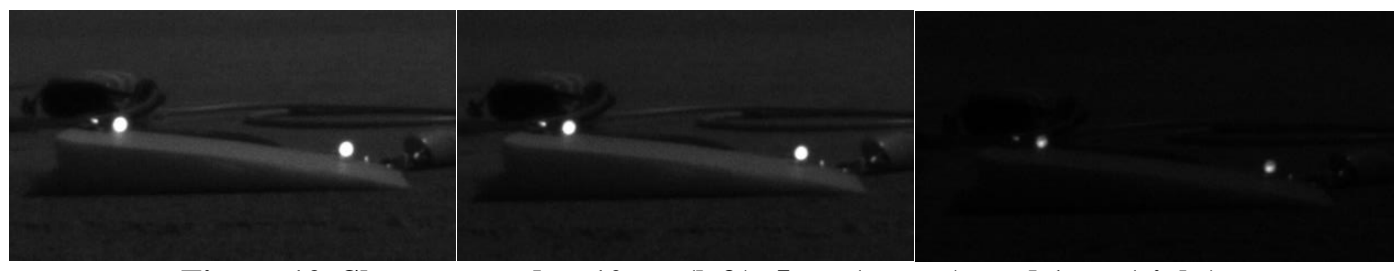

Figure 12. Shutter speed at $10 \mathrm{~ms}$ (left), $5 \mathrm{~ms}$ (center), and $1 \mathrm{~ms}$ (right)

In addition, this test served as a validation test of the light reflection problem on the wing surface. A light dusting of matt white paint was applied to reduce wing reflection and this significantly reduces the LED reflection (Figure 13).

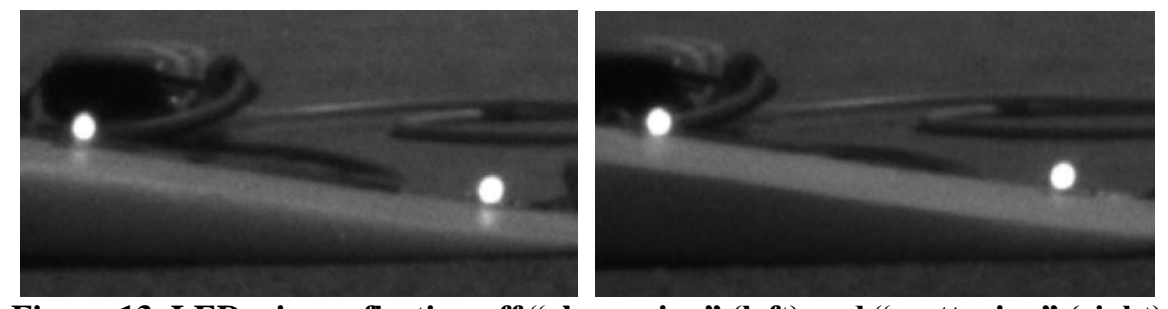

Figure 13. LED wing reflection off "clean wing" (left) and "matt wing" (right)

\section{Concluding Remarks}

This paper presented the hardware and software implementation of a stereovision wing measurement system for small UAV application. Benchmark tests were conducted and the system performance evaluated. The stereovision is capable of less than $5 \mathrm{~mm}$ error at 2-m range for checkerboard targets and less than $7 \mathrm{~mm}$ error for LED targets. For a successful stereovision setup, care must be taken to prevent field-of-view issues and stray light reflections. Wing shape recovery technique using numerical optimization was also discussed.

\section{Acknowledgements}

This work has been supported in part by the Air Force Research Laboratory under the Michigan/AFRL Collaborative Center in Aeronautical Sciences (MACCAS). Additional funds were also provided by the University of Michigan's Active Aeroelasticity and Structures Research Laboratory. The first author would like to thank DSO

11

American Institute of Aeronautics and Astronautics 
National Laboratories, Singapore for the scholarship to pursue graduate studies at the University of Michigan. The authors would also like to thank Dylan Davis, Jessica Jones and the other members of the X-HALE team for their support, as well as Aaron Borgman (University of Michigan) for his aid with the electronics and instrumentation.

\section{References}

${ }^{1}$ Noll, T. E., Brown, J. M., Perez-Davis, M. E., Ishmael, S. D., Tiffany, G. C., and Gaier, M., Investigation of the Helios Prototype Aircraft Mishap. Volume 1: Mishap Report, NASA, Jan 2004.

${ }^{2}$ Su, W., and Cesnik, C. E. S., "Dynamic Response of Highly Flexible Flying Wings," AIAA Journal, Vol. 49, No. 2 , 2011, pp. 324-339.

${ }^{3}$ Cesnik, C. E. S., Senatore, P. J., Su, W., Atkins, E. M., and Shearer, C. M., "X-HALE: A Very Flexible Unmanned Aerial Vehicle for Nonlinear Aeroelastic Tests," AIAA Journal, Vol. 50, No. 12, 2012, pp. 2820-2833.

${ }^{4}$ Moeslund, T. B., and Granum, E., "A Survey of Computer Vision-Based Human Motion Capture," Computer Vision and Image Understanding, Vol. 81, No. 3, 2001, pp. 231-268.

${ }^{5}$ Desouza, G. N., and Kak, A. C., "Vision for Mobile Robot Navigation: A Survey," IEEE Transactions on Pattern Analysis and Machine Intelligence, Vol. 24, No. 2, 2002, pp. 237-267.

${ }^{6}$ Brown, M. Z., Burschka, D., and Hager, G. D., "Advances in Computational Stereo," IEEE Transactions on Pattern Analysis and Machine Intelligence, Vol. 25, No. 8, 2003, pp. 993-1008.

${ }^{7}$ Burner, A. W., and Liu, T., "Videogrammetric Model Deformation Measurement Technique," Journal of Aircraft, Vol. 38, No. 4, 2001, pp. 745-754.

${ }^{8}$ Lamiscarre, B. B., Sidoruk, B., Selvaggini, R., Castan, E., and Bazin, M., "Stereo-Optical System for HighAccuracy and High-Speed 3D Shape Reconstitution: Wind-Tunnel Applications for Model Deformation Measurements," SPIE Proceedings, edited by Kyrala, G. A., and Snyder, D. R., SPIE, San Diego, California, 1994, pp. 46-55.

${ }^{9}$ Albertani, R., Stanford, B., Hubner, J. P., and Ifju, P. G., "Aerodynamic Coefficients and Deformation Measurements on Flexible Micro Air Vehicle Wings," Experimental Mechanics, Vol. 47, No. 5, 2007, pp. 625-635.

${ }^{10}$ Burner, A., Fleming, G., and Hoppe, J., "Comparison of Three Optical Methods for Measuring Model Deformation," 38th Aerospace Sciences Meeting and Exhibit, American Institute of Aeronautics and Astronautics, Reston, Virigina, 2000.

${ }^{11}$ DeAngelis, V. M., "In-flight Deflection Measurement of the HiMAT Aeroelastically Tailored Wing," Journal of Aircraft, Vol. 19, No. 12, 1982, pp. 1088-1094.

${ }^{12}$ Allen, M., Lizotte, A., Dibley, R., and Clarke, R., "Loads Model Development and Analysis for the F/A-18 Active Aeroelastic Wing Airplane," AIAA Atmospheric Flight Mechanics Conference and Exhibit, AIAA, Reston, Virigina, 2005.

${ }^{13}$ Kurita, M., Koike, S., Nakakita, K., and Masui, K., "In-Flight Wing Deformation Measurement," 51st AIAA Aerospace Sciences Meeting Including The New Horizons Forum and Aerospace Exposition, AIAA, Grapevine, Texas, 2013, pp. 1-7.

${ }^{14}$ Kirby III, G. C., Lim, T. W., Weber, R., Bosse, A., Povich, C., and Fisher, S., "Strain-Based Shape Estimation Algorithms for a Cantilever Beam," Proc. SPIE 3041, Smart Structures and Materials 1997: Smart Structures and Integrated Systems, edited by Regelbrugge, M. E., SPIE, 1997, pp. 788-798. 
${ }^{15}$ Kirby III, G. C., Lindner, D. K., Davis, M. A., and Kersey, A. D., "Optimal Sensor Layout for Shape Estimation from Strain Sensors," Proc. SPIE 2444, Smart Structures and Materials 1995: Smart Sensing, Processing, and Instrumentation, edited by Spillman, Jr., W. B., SPIE, San Diego, California, 1995, pp. 367-376.

${ }^{16}$ Kang, L.-H., Kim, D.-K., and Han, J.-H., "Estimation of Dynamic Structural Displacements Using Fiber Bragg Grating Strain Sensors," Journal of Sound and Vibration, Vol. 305, No. 3, 2007, pp. 534-542.

${ }^{17}$ Ko, W. L., Richards, W. L., and Tran, V. T., Displacement Theories for In-Flight Deformed Shape Predictions of Aerospace Structures. NASA/TP-2007-214612, Edwards, California, 2007.

${ }^{18}$ Parker, A., Richards, L., and Ko, W., "Fiber Bragg Grating Sensor / Systems for In-Flight Wing Shape Monitoring of Unmanned Aerial Vehicles ( UAVs )," Sensors GOV Expo Conference, NASA, Hampton, Virginia, 2005.

${ }^{19}$ Richards, L., Parker, A. R., Ko, W. L., and Piazza, A., "Real-Time In-Flight Strain and Deflection Monitoring with Fiber Optic Sensors," Space Sensors and Measurements Techniques Workshop, Nashville, Tennessee, August 2008. Available: http://www.nasa.gov/offices/ipp/centers/dfrc/technology/DRC-006-024-FiberShapeSensing.html $(12 / 12 / 2013)$.

${ }^{20}$ Zhang, Z., "A Flexible New Technique for Camera Calibration," IEEE Transactions on Pattern Analysis and Machine Intelligence, Vol. 22, No. 11, 2000, pp. 1330-1334.

${ }^{21}$ Heikkila, J., and Silven, O., "A Four-Step Camera Calibration Procedure with Implicit Image Correction," Proceedings of IEEE Computer Society Conference on Computer Vision and Pattern Recognition, IEEE Comput. Soc, Washington, DC, 1997, pp. 1106-1112.

${ }^{22}$ Bradski, G. R., and Kaehler, A., Learning OpenCV: Computer Vision with the OpenCV Library, 1st ed., O'Reilly Media, Sebastopol, California, 2008, p. 555.

${ }^{23}$ Marquardt, D. W., “An Algorithm for Least-Squares Estimation of Nonlinear Parameters," Journal of the Society for Industrial and Applied Mathematics, Vol. 11, No. 2, 1963, pp. 431-441.

${ }^{24} \mathrm{Su}$, W., and Cesnik, C. E. S., "Strain-Based Geometrically Nonlinear Beam Formulation for Modeling Very Flexible Aircraft," International Journal of Solids and Structures, Vol. 48, No. 16-17, 2011, pp. 2349-2360. 